\title{
Las nuevas leyes de los nuevos medios y la reconfiguración del entorno
}

\author{
Fernando Gutiérrez-Cortés ${ }^{1}$ \\ Octavio Islas-Carmona ${ }^{2}$ \\ Amaia Arribas-Urrutia ${ }^{3}$
}

Recibido: 16/01/2018

Aprobado por pares: 21/03/2018
Enviado a pares: 07/02/2018

Aceptado: 09/04/2018

DOI: $10.5294 /$ pacla.2019.22.2.9

Para citar este artículo / to reference this article / para citar este artigo Gutiérrez-Cortés, F., Islas-Carmona, 0. y Arribas-Urrutia, A. (2019). Las nuevas leyes de los nuevos medios y la reconfiguración del entorno. Palabra Clave, 22(2), e2229.

DOI: 10.5294/pacla.2019.22.2.9

\section{Resumen}

Este artículo tiene el objetivo de analizar de manera reflexiva y analítica la relación existente entre las tecnologías de la información y de la comunicación (TIC) y la reconfiguración del entorno en el imaginario de la ecología de medios. Para ello, la metodología utilizada es la revisión teórica de conceptos como la exaptación de la biología evolutiva pasando por el desarrollo y evolución de los medios y las tecnologías, que destaca la importancia del trinomio ciencia-tecnología-sociedad. Las TIC facilitan el proceso de exaptación en las sociedades contemporáneas, favoreciendo que las personas descubran nuevas posibilidades de desarrollo y nuevas formas de conocimiento. Desde la década de los sesenta, se subrayó la importancia del estudio de los medios como instrumentos con características singulares, ya que afectan a la sociedad por su diseño, forma y estructura. Cuando una tecnología, medio o instrumento irrumpe exitosamente en una sociedad, la redefine profundamente. Pero el proceso de adaptación no es suficiente para explicar la complejidad de los cambios tecnológicos. Así, el concepto

\footnotetext{
orcid.org/0000-0003-1753-3527. Tecnológico de Monterrey, México. fgutierr@itesm.mx

2 orcid.org/0000-0002-6562-3925. Pontificia Universidad Católica de Ecuador, Ecuador. joislas@puce.edu.ec

3 orcid.org/0000-0001-9452-8364. Universidad de Los Hemisferios, Ecuador. amayaa@uhemisferios.edu.ec
} 
de exaptación incorporado al imaginario de la ecología de medios ayuda a entender la transformación evolutiva de los seres y las tecnologías más allá de sus ambientes específicos. La aportación original de este artículo radica en la incorporación del concepto de exaptación al imaginario teórico y conceptual de la comunicología, y muy concreto a la ecología de los medios. De esta manera, la exaptación permite evaluar la pertinencia de las leyes de los medios de la tétrada de Marshall y Eric McLuhan.

\section{Palabras clave (fuente: tesauro de la Unesco)}

Exaptación; ecología de los medios; Marshall McLuhan; tecnologías de la información y de la comunicación; TIC; impacto de la comunicación; cambio de actitud. 


\section{The New Laws of New Media and the Re-shaping of the Environment}

\section{Abstract}

This article aims to reflectively and analytically explore the relationship between information and communication technologies (ICT) and the reshaping of the environment in the imaginary of media ecology. For this purpose, the method used is the theoretical review of concepts such as exaptation from evolutionary biology, passing through the development and evolution of media and technologies and highlighting the importance of the science-technology-society trinomial. ICTs facilitate the process of exaptation in contemporary societies, encouraging individuals to discover new possibilities for development and new forms of knowledge. Since the sixties, the importance of the study of media as instruments with unique characteristics has been underlined since they affect society for their design, form and structure. When a technology, medium or instrument successfully breaks into a society, it redefines it deeply. But the process of adaptation is not enough to explain the complexity of technological changes. Therefore, the concept of exaptation incorporated into the imaginary of media ecology helps to understand the evolutionary transformation of beings and technologies beyond their specific environments. The original contribution of this article lies in the incorporation of the concept of exaptation to the theoretical and conceptual imaginary of communicology, specifically media ecology. In this way, exaptation makes it possible to assess the relevance of Marshall and Eric McLuhan's tetrad of media effects.

\section{Keywords (source: Unesco Thesaurus)}

Exaptation; media ecology; Marshall McLuhan; information and communications technology; ICT; communication impact; attitude change. 


\section{As novas leis das novas mídias e a reconfiguração do entorno}

\section{Resumo}

Este artigo tem como objetivo analisar de forma reflexiva e analítica a relação entre as tecnologias da informação e comunicação (TIC) e a reconfiguração do entorno no imaginário da ecologia dos meios de comunicação. A metodologia utilizada foi a revisão teórica de conceitos, como a exaptação da biologia evolutiva através do desenvolvimento e a evolução de mídias e tecnologias, o que evidencia a importância do trinômio ciência-tecnologiasociedade. As TIC facilitam o processo de exaptação nas sociedades contemporâneas, incentivando as pessoas a descobrirem novas possibilidades de desenvolvimento e novas formas de conhecimento. A importância do estudo dos meios de comunicação como instrumentos com características únicas tem sido destacada, desde os anos 1960, já que afetam a sociedade a partir da sua concepção, forma e estrutura. Quando uma tecnologia, meio ou instrumento entra com sucesso em uma sociedade tem o poder de redefini-la profundamente. Porém, o processo de adaptação não é suficiente para explicar a complexidade das mudanças tecnológicas. Sendo assim, o conceito de exaptação incorporado ao imaginário da ecologia dos meios de comunicação ajuda a entender, muito além dos ambientes específicos, a transformação evolutiva dos seres e das tecnologias. A contribuição original deste artigo está na incorporação do conceito de exaptação ao imaginário teórico e conceitual da comunicologia e, concretamente, à ecologia dos meios de comunicação. Dessa forma, a exaptação torna possível avaliar a relevância das leis dos meios de comunicação da tétrade de Marshall e Eric McLuhan.

\section{Palavras-chave (Fonte: tesauro da Unesco)}

Exaptação; ecologia dos meios de comunicação; Marshall McLuhan; novas tecnologias de informação e comunicação; NTIC; impacto da comunicação; mudança de atitude. 


\section{Introducción}

En 1964, Herbert Marshall McLuhan, quien realizó estudios doctorales en literatura inglesa en la Universidad de Cambridge, publicó su cuarto libro Understanding media The extensions of man (Comprender los medios de comunicación: las extensiones del ser humano), el cual lo convirtió en uno de los íconos de la década de los sesenta y del siglo XX, como afirman sus principales biógrafos (Carey, 1967; Duffy, 1969; Gordon, 1988; Marchand, 1989; Miller, 1971). En el citado texto, el destacado filósofo de la comunicación subrayó la importancia del estudio de los medios como objetos o artefactos con características singulares, los cuales afectan a la sociedad, no tanto por su contenido, sino por su diseño, forma y estructura. Bajo la lógica del afamado profesor canadiense, cuando una tecnología, medio, instrumento o artefacto irrumpe exitosamente en una sociedad, la redefine profundamente. Así como el cine, la radio y la televisión contribuyeron a modificar las percepciones y los hábitos de las personas en el siglo pasado, de modo similar las nuevas tecnologías de comunicación digital están reconfigurando la cultura de las sociedades en esta nueva era, designada por Toffler y Toffler (2006) como la "economía del conocimiento" y por McLuhan (1964) como information age (edad de la información).

El tránsito a la edad poseléctrica ha implicado profundos cambios. El proceso de adaptación social, dadas las nuevas y complejas exigencias del entorno, de ninguna manera resulta sencillo. En las primeras líneas de The medium is the massage: An inventory of Effects (1967), quinto libro de McLuhan, el cual fue ilustrado por el reconocido diseñador gráfico Quentin Fiore, describió la magnitud de los cambios que depararía el tránsito a una nueva época:

The medium, or process, of our time -electric technology - is reshaping and restructuring patterns of social interdependence and every aspect of our personal life. It is forcing us to reconsider and re-evaluate practically every thought, every action and every institution formally taken for granted. Everything is changing -you, your family, your neighborhood, your education, your job, your government, your relation to "the others". And they're changing dramaticaIly. ${ }^{4}$ (McLuhan, 1967, pp. 8-9)

4 El medio, o proceso, de nuestra era - la tecnología eléctrica - está remodelando y reestructurando los patrones de interdependencia social y cada aspecto de nuestra vida personal. Nos obliga a reconsiderar y reevaluar prácticamente 
McLuhan, quien en no pocas ocasiones ha sido etiquetado por sus detractores como uno de los pensadores emblemáticos del "tecnooptimismo", con notable claridad advirtió los efectos que producen las tecnologías en el desarrollo cultural de las sociedades. ${ }^{5}$ Si entendemos las revolucionarias transformaciones causadas por las tecnologías (razonaba), podremos anticiparlas, controlarlas y adaptarnos; pero, si continuamos en nuestro trance subliminal, siempre seremos esclavos de los efectos tecnológicos.

Sin embargo, es importante tener presente que el proceso de adaptación no es suficiente para explicar la complejidad de los cambios tecnológicos. En On the origin of species by means of natural selection (1859), Darwin destacó que para que una especie resienta alguna modificación importante, un órgano ya formado tiene que variar de nuevo. Gould (1991) designó ese complejo proceso como exaptación, y usó ese término para referirse a toda aquella característica, presente en una especie, que originalmente cumplía un rol distinto del que actualmente desempeña. En este artículo, incorporaremos el concepto exaptación al imaginario teórico y conceptual de la comunicología, y muy en particular a la ecología de los medios. La exaptación nos permitirá evaluar la pertinencia de las leyes de los medios que presentaron Marshall y Eric McLuhan con su "tétrada”.

\section{El valor de la exaptación como factor de innovación conveniente}

Podemos definir la exaptación como un complejo proceso de evolución. Se parte de un rasgo original, el cual favorece la adaptación a determinadas condiciones; pero, una vez que la adaptación realizada se ha consolidado, esta evoluciona para responder a otras finalidades (Gould, 1991). Un ejemplo de ello ocurrió con las plumas de las aves, las cuales, primero, les permitieron regular la temperatura, pero que con el paso del tiempo evolucionaron hasta permitir la realización de una función mucho más compleja: volar.

cada pensamiento, cada acción y cada institución formalmente dada por sentada. Todo está cambiando: usted, su familia, su vecindario, su educación, su trabajo, su gobierno, su relación con "los otros”. Y están cambiando drásticamente.

5 Entendemos la ecología de los medios como una compleja metadisciplina que se encarga de estudiar los efectos que producen las tecnologías en las sociedades a lo largo de la historia.

6 Las nuevas leyes de los nuevos medios y la reconfiguración del entorno - Fernando Gutiérrez Cortés y otros 
El concepto exaptación ha trascendido las fronteras teórico-conceptuales de la biología evolutiva. En la ecología de los medios, ha sido recuperado para emprender el análisis de tecnologías como la imprenta, el telégrafo, el teléfono, la radio, la televisión e internet, los cuales han producido drásticos cambios en las sociedades. La imprenta de Johannes Gutenberg, por ejemplo, no es el resultado de una adaptación sino de una exaptación de las prensas utilizadas para exprimir el jugo de uva en la elaboración del vino. Gutenberg experimentó con artefactos que ya existían, como el tipo móvil y la prensa vinícola, hasta conseguir la imprenta mecánica (Rees, 2006). Algo similar ocurrió con otras tecnologías de comunicación, las cuales han sido el resultado de "exaptaciones" que fueron posibles gracias a ciertas condiciones del entorno, así como a la formidable visión de sus desarrolladores. Tal es el caso de la imprenta (Johannes Gutenberg), el telégrafo (Pavel Schilling y Samuel Morse), el teléfono (Antonio Meucci y Alexander Graham Bell), la radio (Guglielmo Marconi, Aleksandr Stepánovich Popov, Nikola Tesla, Julio Cervera), la televisión (John Logie Baird), internet (Vinton Cerf) y la World Wide Web (Tim Berners Lee). Cada uno de los desarrolladores de los referidos medios tomó algo de lo que ya existía, procediendo a utilizarlo de manera distinta y para otro propósito. En Mind amplifier: Can our digital tools make us smarter? (2013), Rheingold destaca que la exaptación puede ser entendida como un factor de innovación conveniente. Las principales innovaciones que se han presentado durante el siglo XXI son el resultado de exaptaciones que se han manifestado en el entorno digital. Los creadores de nuevas y exitosas plataformas tecnológicas han seguido - y probablemente sin saberlo- la propuesta de Rheingold. Han tomado algo que ya existía para construir otra cosa distinta, susceptible de ser utilizada para otros propósitos. La exaptación los ha llevado a un nivel de innovación compleja. Mientras la adaptación cumple útiles funciones que permiten la supervivencia de una especie en un entorno determinado, la exaptación contribuye a la gran transformación evolutiva de los seres y las tecnologías más allá de sus ambientes específicos (Gould y Vrba, 1982). El concepto de exaptación es complejo, pues es superior al de la adaptación y resulta necesario para asegurar el desarrollo de un sistema determinado en un entorno dinámico; por ende, resulta susceptible de ser recuperado en el imaginario de la teoría de sistemas, el pensamiento complejo y la semántica general. 


\section{Ciencia, tecnología y sociedad: el trinomio perfecto}

En Ciencia, tecnología e historia, Ordóñez (2003) afirmó que suele olvidarse que la ciencia y la tecnología son productos culturales que influyen y que son influidos por el contexto social; sin embargo, los tratamos con excesivo respeto. La historia nos ha enseñado que la ciencia y la tecnología no siempre han estado juntas. Han transitado por senderos separados. La tecnología ha sido una constante en las sociedades humanas, la ciencia no. De hecho, las primeras civilizaciones se caracterizaron por ser sociedades fundamentalmente tecnológicas, y en segundo término, científicas. A lo largo de la historia, la alianza entre ciencia y tecnología ha sido incompleta. La tecnología siempre ha estado presente en la historia de la humanidad, desde la creación del alfabeto fonético, como destaca Logan (2004), y se ha desarrollado con relativa independencia de la ciencia. La ciencia ha aprovechado la tecnología, particularmente cuando requiere mayor precisión. Tal aseveración puede verificarse, por ejemplo, con el experimento realizado por Eddington (1987), astrofísico británico, quien en 1919 confirmó la teoría de la relatividad de Einstein, utilizando equipo fotográfico. En otros casos, la tecnología también ha sido clave para la comprobación de teorías que dieron lugar a nuevas formas de conocimiento. Por ejemplo, el telescopio (tecnología) posibilitó el estudio de los astros, favoreció la indispensable separación de la astronomía (ciencia) de la astrología y permitió la comprobación empírica de diversas teorías sobre el espacio. Desde las observaciones de Galileo, en el siglo XVII, hasta nuestros días, el telescopio ha permitido verificar diversas hipótesis sobre el origen y desarrollo del universo. Similar situación se presentó con otras tecnologías como el microscopio, las primeras antenas radioeléctricas, las computadoras y otros artefactos que se han desarrollado a lo largo del tiempo. Por lo anterior, Ordóñez (2003) afirma que la ciencia experimental —aquella que está relacionada con la naturaleza - tiene necesidad de una especie de referencia tecnológica, y no necesariamente al contrario.

Durante el siglo XIII fue desarrollada la primera máquina de vapor, como resultado de un proceso tecnológico y no científico. Entonces nadie conocía las leyes que regulan el movimiento de las máquinas de vapor. 
La mayoría de los ingenieros franceses consideraron la máquina hidráulica como la tecnología del futuro y no la máquina de vapor, porque sencillamente no comprendían el funcionamiento de esta. Ordóñez (2003) sostiene que la comunidad científica francesa parecía privilegiar lo científico sobre lo tecnológico. El desarrollo de las máquinas de vapor alteraría el desarrollo de las ciencias y transformaría las condiciones de vida de millones de personas. Sobre la trascendencia de las máquinas de vapor, Ordoñez afirma que "los telescopios se perfeccionaron gracias a los estudios teóricos de la óptica, pero nuestra comprensión de la naturaleza se perfeccionó gracias a la evolución de las máquinas de vapor” (p. 74).

Debemos destacar que en la relación ciencia-tecnología no se puede considerar la superioridad de una sobre la otra; es decir, la ciencia no puede estar por encima de la tecnología, o viceversa; simplemente, debemos reconocer que cada una se ha desarrollado de forma relativamente diferente a lo largo de la historia. Esta relación ha sido dinámica y, por supuesto, compleja. Sin embargo, de poco han servido la ciencia y la tecnología cuando han resultado indiferentes al contexto social. La sociedad es el factor indispensable para lograr el trinomio perfecto. La ciencia y tecnología deben responder a las exigencias del contexto social para producir verdadero valor. Hasta la fecha las relaciones entre la sociedad, la ciencia y la tecnología han sido la historia de una alianza incompleta (Ordóñez, 2003). En no pocas ocasiones, el desarrollo de la ciencia y la tecnología se han apartado del contexto social. Ante los umbrales de la cuarta revolución industrial, resulta indispensable considerar el mencionado trinomio para efectivamente poder propiciar mejores condiciones de vida en el mundo.

Un ejemplo de la indispensable armonía que debe prevalecer entre ciencia, tecnología y sociedad lo representan las llamadas leyes de la robótica, definidas por Asimov en el relato Runaround (1942). Las referidas leyes son "formulaciones matemáticas impresas en los senderos positrónicos ${ }^{6}$ del cerebro de los robots", y que en todo momento y lugar deben regular su creación y desarrollo. Las tres leyes son estas: a) un robot no hará daño a un ser humano o por inacción permitir que un ser humano sufra daño;

6 Son las líneas de código de programa que regulan el cumplimiento de las leyes guardado en la memoria principal. 
b) un robot debe realizar las órdenes dadas por los seres humanos, excepto si estas órdenes entrasen en conflicto con la primera ley, y c) un robot debe proteger su propia existencia en la medida en que esta protección no entre en conflicto con la primera o la segunda ley. Vale la pena señalar que, de las leyes propuestas por Asimov, derivó la llamada ley cero de la robótica (zeroth law): "Un robot no hará daño a la humanidad y no permitirá que la humanidad sufra daño”.

\section{Aprendizajes derivados de transformaciones en sistemas interrelacionados}

De acuerdo con Christopherson (1994), un ecosistema está formado por organismos (entidades) interdependientes (relacionados) que comparten un mismo medio físico (entorno). En cada ecosistema, se establece siempre una clase de equilibrio dinámico que explica los diferentes tipos de relaciones que desarrollan las diversas entidades de un sistema dentro de un entorno determinado. Cualquier alteración en el equilibrio del sistema se produce por algún cambio en la entidad o en las relaciones que se mantienen en un espacio y tiempo específico. La ecología es una rama de la biología que estudia las interrelaciones entre los diferentes organismos de un sistema y su entorno. A principios de la década de los setenta, Neil Postman, destacado catedrático de la Universidad de Nueva York, promovió el concepto media ecology, el cual tomó de McLuhan para explicar —a través de una analogía con la ecología como rama de la biología — cómo todos los medios o tecnologías de comunicación (entidades) afectan la percepción, la comprensión, los sentimientos y los valores de las personas (relaciones), y cómo esta interacción con las tecnologías aumenta o reduce nuestras posibilidades de supervivencia en un espacio (entorno) y tiempo determinado (Postman, 1970). La ecología de los medios, una metadisciplina, de acuerdo con Nystrom (1973), discípula de Postman, dio origen a un posgrado en la Universidad de Nueva York, el cual formó parte de la oferta curricular de esa universidad durante más de treinta años. Para Postman, la palabra ecología supone el estudio de los ambientes mediáticos o tecnológicos (libros, fotografía, cine, radio, televisión, computadoras); específicamente, de su forma, estructura, contenido e impacto social. En estos ambientes, se presenta un sistema dinámico y complejo de mensajes que condicionan la 
forma de pensamiento, sentimientos y actuaciones de las personas. Por una parte, estructuran lo que se puede ver $y$ decir, establecen roles y especifican lo que se permite y no se permite realizar. En el caso de los ambientes tecnológicos, las especificaciones son cada vez más implícitas e informales, y lo que busca esta compleja metadisciplina - la ecología de los medios- es volver explícitas esas condiciones. La ecología de los medios promueve el estudio de los ambientes mediáticos y explora las consecuencias culturales de las transformaciones tecnológicas en el tiempo, es decir, cómo cambian y nos cambian continuamente los medios, las herramientas, los instrumentos, los artefactos o las tecnologías que se encuentran a nuestra disposición. Tal como anticipó McLuhan (1994) en Understanding media: "We shape our tools and thereafter our tools shape us" (damos forma a nuestras herramientas y luego nuestras herramientas nos dan forma).

\section{Dualidades en el cambio tecnológico}

Un nuevo medio no solo agrega algo a la cultura, sino que la cambia por completo (Postman, 1993). Cuando una nueva tecnología adquiere importancia en una cultura determinada, ciertos elementos que definían esa cultura serán reconfigurados. La nueva cultura resultante es, en gran medida, producto del cambio tecnológico que aparentemente tiene consecuencias impredecibles, rápidas e irreversibles. Mucho se ha hablado de las ventajas que ofrecen las tecnologías en diferentes ámbitos, como la educación, la política, la salud, por mencionar algunos, pero poco suele decirse de las facturas que dejan todas las transformaciones tecnológicas en las sociedades. Los cambios tecnológicos muestran una dualidad de funciones. Por una parte, la tecnología promete siempre, y la mayoría de las ocasiones efectivamente otorga, una serie de beneficios a los usuarios; por otra parte, despoja de ciertas capacidades o funciones a las personas. Lo anterior precisamente fue contemplado en las primeras dos leyes de la tétrada de los McLuhan. Las tecnologías extienden, pero también amputan.

En sus últimos años de vida, McLuhan desarrolló una rigurosa metodología para estudiar el impacto de las tecnologías en las sociedades a lo largo de la historia. En ello colaboró Eric, el mayor de los seis hijos que procreó Marshall. Como Marshall falleció el 31 de diciembre de 1980, Eric se 
dedicó a terminar un libro, en el cual fue incluida la tétrada de los McLuhan, Laws of media: The new science, cuya primera edición fue publicada en 1988. En un texto publicado en 2016, Eric reveló que este libro debe ser considerado como la continuación de Understanding media.

Las cuatro leyes de la tétrada admiten ser aplicadas, sin excepción, a todas las creaciones del hombre, tangibles como intangibles, abstractas o concretas. La tétrada, además, permite confirmar que las tecnologías y los medios pueden prolongar los órganos y las funciones humanas. Las tecnologías mecánicas sirven para extender nuestro cuerpo físico, mientras que los medios de comunicación extienden nuestra psique o el sistema nervioso. Con la tétrada, Marshall y Eric McLuhan pretendieron ofrecer respuesta a las siguientes preguntas: ¿qué afirmaciones podemos hacer acerca de los medios de información que cualquiera pueda poner a prueba - confirmar o refutar-? y zqué tienen en común todos los medios informativos? La figura 1 corresponde a la representación gráfica de la tétrada de los McLuhan, la cual no debe ser considerada secuencialmente, pues se trata de cuatro procesos más o menos simultáneos.

\section{Figura 1. Tétrada de los McLuhan \\ Objeto de análisis}

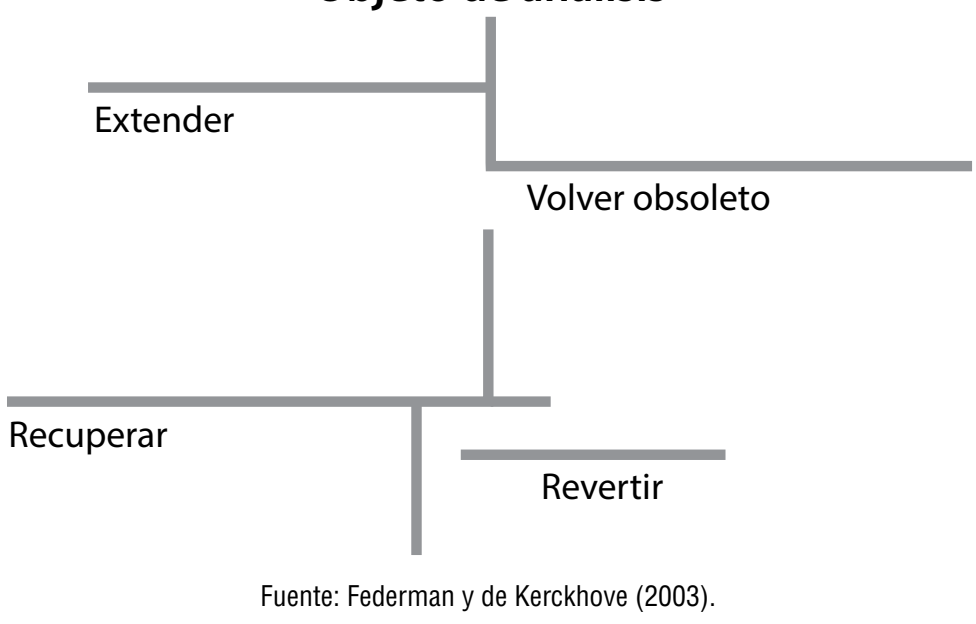

La primera ley, relativa a la extensión, indica que toda tecnología extiende una facultad física o psíquica del hombre. El concepto de exten- 
sión supone la posibilidad de prolongar, reemplazar, incrementar, reforzar, acelerar, intensificar. Cada medio, tecnología o artefacto elaborado por el hombre mejora alguna función humana. La segunda ley corresponde a la obsolescencia y es consecuencia directa de la extensión. Ello implica que, cuando un medio prolonga una facultad física o psíquica, determinadas partes del entorno de lo extendido se vuelven obsoletas. Las tecnologías extienden, pero también amputan. La amputación es inevitable. Mientras determinados sentidos o facultades se incrementan, otros inevitablemente disminuyen. Toda nueva tecnología vuelve obsoletas tecnologías precedentes. McLuhan comprendía la obsolescencia no como el final, sino como el comienzo de algo nuevo. El concepto medular de la tercera ley es la recuperación. La estructura social resiente los efectos de toda nueva tecnología, y paulatinamente recupera su equilibrio. En ocasiones, la recuperación es resultado de adaptaciones o procesos compensatorios. La cuarta ley se define a partir de la reversión. Cuando la tecnología llega a su límite (que en términos mcluhianos significa el sobrecalentamiento del medio), suelen presentarse hechos y situaciones que revierten o "enfrían" la tendencia. Un medio solo se revierte porque se ha llevado al límite.

Centraremos nuestra atención en las dos primeras leyes. En la primera (relativa a la extensión), se considera, en términos generales, que cualquier tecnología de comunicación extiende o amplifica una facultad física o psíquica del hombre. En la segunda ley, denominada de la obsolescencia, se plantea que un nuevo medio o tecnología puede disminuir una capacidad o facultad humana. Al mismo tiempo que algo se extiende, otra capacidad o facultad disminuye, e inclusive puede volverse obsoleta. En esta especie de pacto, la tecnología siempre otorga, pero también quita (Postman denominó ese proceso como "el pacto de Fausto"), y cuando este fenómeno se produce, las personas no lo perciben porque experimentan una especie de hipnosis que les impide percatarse del proceso. En la actualidad, internet, por ejemplo, ha modificado radicalmente el concepto de privacidad (particularmente en los consumidores). Al efectuar compras en un sitio web, los usuarios contribuyen a la gestación y el desarrollo de patrones de consumo, los cuales pueden ser registrados por un sistema inteligente (big data), que aprende a partir de las preferencias de los clientes. Por esa razón, muchas 
personas suelen asombrarse cuando alguna tienda virtual les recomienda algún producto o servicio que efectivamente coincide con sus preferencias. En la era de internet, el concepto de privacidad debe ser redefinido.

El cambio tecnológico desplaza la introducción de nuevos productos y servicios a la sociedad, pero también se desprenden consecuencias intencionadas y no intencionadas. Estas surgen, por lo general, en la última etapa de vida del ciclo tecnológico, y resultan ser las más peligrosas, por ser imprevisibles. En torno al proceso de industrialización, con el paso de los años, surgió un particular estilo de vida en las grandes ciudades y sus suburbios. Las ciudades contribuirían a elevar la calidad de vida de las personas. Sin embargo, el espacio urbano hizo evidentes las grandes diferencias que prevalecen entre las clases sociales. La urbanización trajo consigo grandes transformaciones, de las cuales se desprendieron repercusiones negativas. La construcción de grandes avenidas y arterias viales agilizó el tránsito vehicular, pero incrementó la contaminación del medio ambiente. En un principio, la mayoría de las personas destacaba los beneficios obtenidos por la reducción de tiempos en sus desplazamientos cotidianos, sin advertir el grave deterioro causado a la calidad del aire.

Por último, extendamos — como simple divertimento — el imaginario de las leyes de los medios. Otras leyes de los medios de comunicación son espantosamente predictivas. Por ejemplo, la ley de medios de Murphy: "Si algo puede salir mal, con un medio seguro lo hará". Razor eléctrico de Occam: "Si dos medios producen efectos equivalentes en la misma audiencia, entonces el más barato es el mejor (a partir de entonces, el más barato será comprado por Microsoft y no funcionará más"). Ley de conservación de los medios de comunicación: "En un servidor de listas, la cantidad total de nueva información no aumenta ni disminuye, sino que permanece exactamente igual; es decir, su tasa de cambio es cero. No se puede crear información nueva, ni destruir información antigua. Ello no impide que los usuarios vuelvan a empaquetar y reciclarlos una y otra vez". Las leyes termodinámicas de los medios de comunicación: "Primera ley: El cambio en el valor de entretenimiento de la información dentro de un sistema es la suma del calor transferido al sistema o desde el mismo, mientras que no 
está trabajando en nada importante. Segunda ley: La entropía de un sistema - una medida de la indisponibilidad de la energía de un sistema para hacer un trabajo útil — tiende a aumentar en una lista de servidores". Las leyes de los medios de Newton. "Primera ley: Un cuerpo continúa en su estado de velocidad constante hasta que es actuado por una fuerza externa, generalmente internet. Entonces todo se detiene. Segunda ley: En un sistema donde no hay fuerzas externas presentes, Google inventa algo". Principio antrópico de los medios de comunicación: "Las condiciones necesarias para el desarrollo de la vida inteligente solo se cumplirán en ciertas regiones del universo que no tienen televisión. La región del universo en la que vivimos no es necesariamente representativa de un conjunto aleatorio de condiciones iniciales; después de todo, los seres humanos crearon Jersey Shore". La ley de Murphy de las leyes de los medios de comunicación: "Si algo puede salir mal con una ley de medios, lo hará”.

\section{La comprensión ecológica del cambio tecnológico}

Para toda persona resulta importante saber que, cuando una nueva tecnología adquiere importancia en una cultura determinada, comienza a transformarla drásticamente y de una forma muy particular, pero considerando siempre los elementos esenciales que alguna vez la definieron. En este sentido, se puede decir que una sociedad se convierte en un producto complejo del cambio tecnológico. El cambio tecnológico es ecológico, no aditivo. Un nuevo medio no añade algo, lo cambia todo. En 1500, después de que la imprenta fue inventada, no se tenía a la vieja Europa más la imprenta. Se tenía una Europa diferente. Después de la televisión, América no era América más la televisión. La televisión dio una nueva coloración a cada campaña política, a todos los hogares, a todas las escuelas, a todas las iglesias, a todas las industrias, y así sucesivamente (Postman, 1998).

Internet ha propiciado profundos cambios ecológicos en las sociedades contemporáneas. El concepto de oficina convencional ha cambiado por el de oficina móvil; del trabajo en oficinas, hemos pasado al teletrabajo; de la tienda tradicional, a las tiendas virtuales. Tal y como destacaba Harold Innis, colega y mentor de Marshall McLuhan, cuando surge un nuevo me- 
dio de comunicación, se crean nuevos patrones de asociación y se articulan nuevas formas de conocimiento (citado por Carey, 1967). Paulatinamente, se presenta un cambio ecológico que transforma todos los elementos interdependientes de un sistema. Esta transición propicia la redefinición completa de procesos que se configuran a partir del establecimiento de nuevas condiciones en el entorno.

Algunos investigadores de la American Psychological Association (APA) señalan que se culpa a internet de la destrucción de matrimonios, de los problemas que abruman a los menores de edad, del creciente gasto en materia de telecomunicaciones, así como del incremento en las acciones delictivas y fraudulentas. No obstante, sus expertos coinciden en afirmar que internet no es culpable de la supuesta adicción que le atribuyen, sino que a través de este medio se manifiestan patologías preexistentes. Ellos señalan que para entender el verdadero problema se debe observar qué hay detrás de esos comportamientos que se presentan a través de la red (DeAngelis, 2000). Lo anterior es claro ejemplo del cambio ecológico. Las tecnologías asociadas al desarrollo de internet han permitido nuevas formas de comunicación, de almacenamiento y administración de la información y de creación de nuevos servicios. Internet ha aportado a los medios convencionales predecesores tecnologías multimedia (texto, gráficos, imágenes y sonido), interactividad y un entorno de compatibilidad tecnológica de redes y plataformas electrónicas sin precedentes en la historia. En el ámbito comercial, ha propiciado el desarrollo de nuevos canales de difusión y comunicación de alcance masivo y ampliado significativamente las posibilidades de negocios. En algunos casos, las tecnologías asociadas al desarrollo de internet han sido las causantes de la ruptura con las empresas intermediarias dentro de una cadena de valor. Sin duda alguna, esas tecnologías digitales asociadas a internet han desempeñado un papel muy importante en la formación de empresas virtuales y favorecido el desarrollo y la venta de nuevos productos o la sustitución de antiguas mercancías por sus equivalentes digitales.

El desafío que hoy enfrentan las naciones está relacionado con la necesidad de acelerar la velocidad de acceso y el uso de internet como herramienta de información, comunicación, comercio y educación, de forma que pueda contribuir a mejorar la calidad de vida a los ciudadanos (véanse los 
reportes realizados por The Broadband Comission for Digital Development sobre la calidad de la banda ancha en el mundo, y los del World Economic Forum relativos a la competitividad mundial de las naciones). Entender cómo la gente se apropia de la tecnología y cómo esta modifica la cultura de las sociedades representa uno de los principales temas de interés en la ecología de los medios.

\section{La nueva ecología digital}

Los medios de comunicación hoy compiten de forma más intensa por la atención de las personas (Levinson, 2004, p. 12). En términos darwinianos, la selección del ambiente mediático queda en manos de las personas, quienes contribuyen con sus preferencias a la evolución de aquellos medios que prefieren. Con frecuencia, decidimos entre ir al cine o quedarnos en casa a ver televisión; leer un libro o ver un video; hablar por celular o enviar correos electrónicos. Los medios no evolucionan por selección natural, sino por una selección humana. El medio que mejor evoluciona es aquel que se ajusta más a las diversas necesidades del hombre. Al respecto, Piscitelli señala:

Los cambios en la tecnología de la comunicación crean efectos sociales, culturales, técnicos y cognitivos potenciales, cuyo nivel de efectividad depende de la naturaleza, alcance y nivel de implementación de la tecnología. Para empezar, ninguna tecnología de la comunicación borra 0 elimina las tecnologías anteriores, pero puede alterar la cantidad de uso de los canales previos y el poder de dominación. (2005, p. 142)

Fidle (1997) anticipó que los medios convencionales resentirían la "mediamorfosis" provocada por la llegada de nuevas tecnologías digitales. La mediamorfosis es el cambio de un medio de comunicación como resultado de las transformaciones que registra la sociedad y el desarrollo de nuevos avances tecnológicos. Ello precisamente ha ocurrido con el teléfono, la radio, el cine y la televisión. En la siguiente infografía (figura 2), realizada por la Interactive Advertising Bureau-México (IAB México, 2017), la cual fue presentada en la novena edición del Estudio de consumo de medios y dispositivos entre internautas mexicanos 2017, podremos advertir algunos de los principales cambios que ha introducido internet en la sociedad mexicana. 


\section{Figura 2. El impacto de internet en la sociedad mexicana}

ESTUDIO DE CONSUMO DE MEDIOS Y DISPOSITIVOS ENTRE INTERNAUTAS MEXICANOS 2016
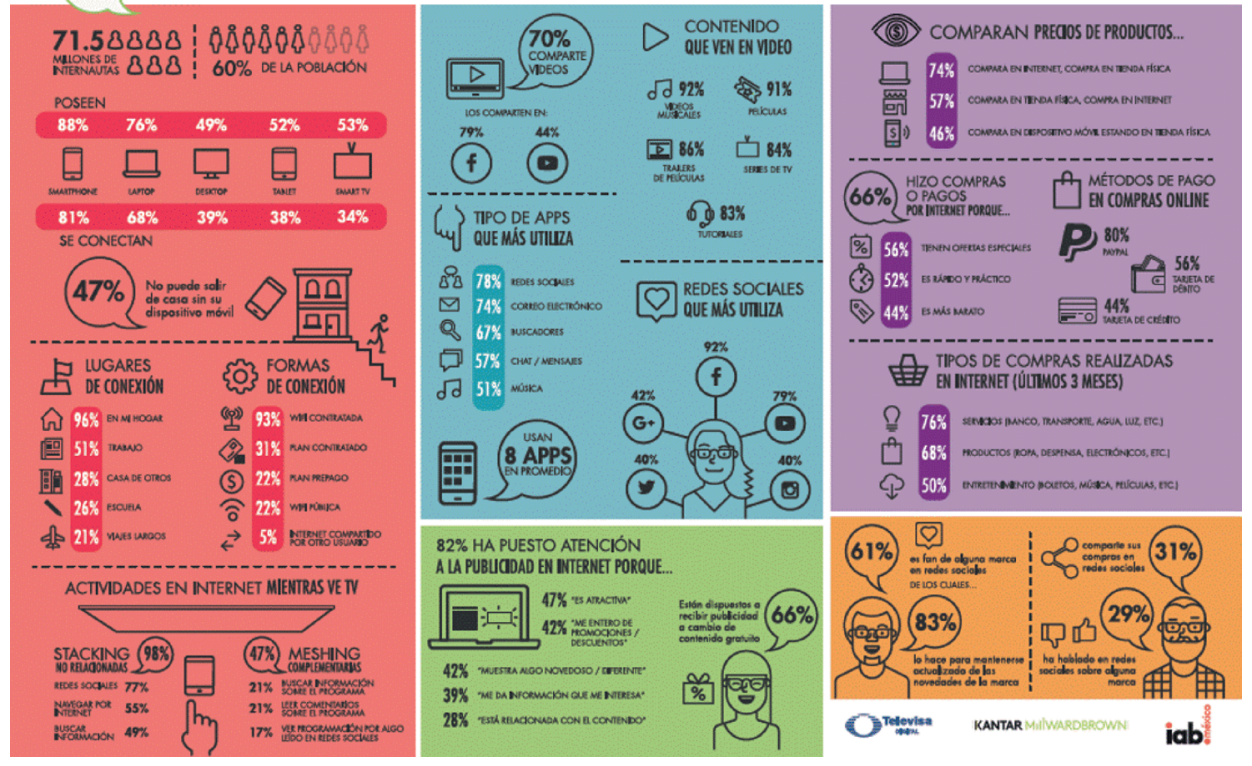

Fuente: IAB México (2017).

En la infografía, podemos apreciar el importante crecimiento que ha tenido internet en México, 71,5 millones de usuarios que representan el $60 \%$ de la población. Por otra parte, destaca cómo los medios convencionales han encontrado una forma de extenderse a través de nuevos dispositivos digitales como móviles, tabletas, laptops y televisiones inteligentes. Además de la información sobre los lugares y las formas de conexión, comienza a sobresalir el tema del meshing. El 47 \% de las personas señalan que mientras ven televisión realizan algunas actividades relacionadas con el contenido que observan mediante sus dispositivos digitales. Ello es propicio para el desarrollo de narrativas transmedia. En la segunda columna de la infografía, se muestra cómo cierto tipo de contenidos resulta mejor para el medio digital que para el convencional. Por otra parte, se presentan los tipos de apps y las redes sociales que más se utilizan y la eficacia de la publicidad digital. Finalmente, la tercera columna nos permite apreciar los usos comerciales que le dan a internet, primero como sistema de referencia para la compa- 
ración de precios de productos y servicios, segundo como medio de compra preferido para cierto tipo de bienes - destacando los medios de pago utilizados - y tercero como medio idóneo para consolidar la merca. Estas cifras demuestran cómo los nuevos medios han introducido nuevos paradigmas en distintos ámbitos de la vida cotidiana.

\section{Extensiones y amplificaciones de los nuevos medios digitales}

Las nuevas tecnologías también pueden amplificar y extender funciones y capacidades de las personas en un entorno determinado. Por tal motivo, las tecnologías pueden ser entendidas como potenciadoras de acción, porque extienden capacidades que estaban limitadas por diferentes circunstancias, y amplifican funciones que se mantenían en cierto estado de equilibrio (Reynolds, 2007; Sánchez de Antuñano, 2014). Para McLuhan (1964), todos los medios son extensiones o amplificaciones de algún sentido, capacidad o función del cuerpo humano. El libro era una extensión de la vista; la radio, una extensión del oído; el teléfono, una extensión del oído y la voz; y la televisión, una extensión del sentido del tacto. Si se asumen como ciertas las tesis contenidas en la tétrada de los McLuhan, todo medio o herramienta es una extensión o amplificación de alguna capacidad o función del cuerpo humano, entonces habría que preguntarse qué es lo que extienden y amplifican las nuevas tecnologías digitales. La tecnología digital cambió drásticamente al mundo. Con el desarrollo de las primeras computadoras fue posible acceder a un nivel de procesamiento de información y cálculo que superaba con creces la capacidad de las personas. Entonces el poder de la mente comenzó a amplificarse, tal y como afirma Rheingold (2013) en Mind amplifier: Can our digital tools make us smarter? Quizá ello represente la mejor respuesta a Nicholas Carr, finalista del Premio Pulitzer en la categoría de "No ficción" con el libro The shallows: What the internet is doing to our brains (2010). Para Carr, la capacidad de concentración en una actividad es muy importante para ejercitar la memoria a largo plazo, desarrollar el pensamiento crítico y conceptual, y generar muchas formas de creatividad. Incluso lo emocional demanda tiempo para ser procesado, y si no se dedica tiempo suficiente para ello, la sociedad se deshumaniza cada vez más. 
El problema es que la revolución digital ha instalado a las sociedades en un ideal muy utilitario, en que lo más importante es la eficiencia en el procesamiento de información. A medida que se cede el control y se externaliza la resolución de problemas y otras tareas cognitivas a las máquinas, el potencial del cerebro se reduce de manera sutil pero significativa. Tal y como sucede cuando un deportista deja de entrenar, su desempeño y rendimiento comienzan a atrofiarse.

Sin embargo, Rheingold (2013) afirma que la ampliación de la memoria, el razonamiento y la capacidad de comunicación han estimulado el desarrollo de una mayor neuroplasticidad en las personas, una especie de reprogramación del cerebro para adaptarse con mayor facilidad al entorno y generar así nuevas formas de pensamiento. Para Rheingold, las computadoras personales son una exaptación de las primeras tecnologías digitales que se desarrollaron en plena Guerra Fría.

La computadora de uso personal es una exaptación cultural de las tecnologías que soñaron los visionarios y financiaron los guerreros desde la década de los cuarenta, y fueron comercializadas en masa por los emprendedores desde la década de los setenta. Una potencia de cálculo que ni siquiera el Departamento de Defensa de los Estados Unidos podía permitirse hace unas décadas y está ahora en las manos de billones de personas (Rheingold, 2013, p. 14).

Los dispositivos conectados a internet son una extensión sumamente versátil de nuestros sentidos, nuestro conocimiento y nuestra memoria, y cumplen importantes funciones como amplificadores neuronales de gran alcance. Sin embargo, algunos sugieren que los efectos negativos de las nuevas tecnologías digitales pueden ser sumamente contraproducentes (Carr, 2011). Por tal razón, debe siempre realizarse una profunda evaluación de cualquier nueva tecnología. Hoy los diversos dispositivos móviles - especialmente los teléfonos inteligentes - funcionan como potentes minicomputadoras. Las nuevas tecnologías digitales nos permiten realizar el trabajo fuera de la oficina o la casa, nos ayudan a estudiar, entretienen y sirven para estar en contacto con miles de personas a través de redes sociales. Aparen- 
temente, no se podría vivir sin estas nuevas tecnologías digitales. Por ejemplo, cuando una persona sale sin su smartphone, siente pánico y algo que los estudiosos del tema han llamado "nomofobia" (no-mobile-phone 'miedo a salir sin el teléfono'). En poco más de treinta años, esta tecnología ha transformado un gran número de hábitos en las personas (Oliver, 2015). Internet, no solo ha extendido las funciones y capacidades del ser humano, sino el poder de los medios de comunicación convencionales, como la prensa escrita, el cine, la radio y la televisión. El smartphone se ha convertido en una extensión del teléfono celular "convencional", la laptop ha derivado de la computadora personal de escritorio, las tabletas o lectores digitales son una evolución de o del periódico impreso. Todas las nuevas tecnologías digitales pueden ser entendidas como una extensión o amplificación de versiones convencionales que les antecedieron. Al respecto, Levinson (1999) afirma que

\begin{abstract}
internet hace contenidos de todos los medios. Aquello que comenzó como un medio cuyo contenido era texto se expandió en la década de los noventa para incluir imágenes y sonidos, y en los umbrales del nuevo milenio ofrece servicios extendidos de telefonía (internet Telephone), radio (RealAudio) y televisión (RealVideo). (p. 5)
\end{abstract}

Hay suficientes evidencias para pensar que las tecnologías digitales seguirán su ciclo de desarrollo, extenderán y amplificarán nuevas funciones y capacidades y, en un momento determinado, evolucionarán a formas más sofisticadas que definitivamente intervendrán en la reconfiguración del ser humano como especie. Sin embargo, esta reconfiguración presenta un riesgo que está relacionado con la pérdida de algunas habilidades fundamentales. De cualquier manera, las tecnologías digitales tienen el potencial de mejorar la calidad de vida y permitir un útil aprovechamiento para el acomodo social a las condiciones de un entorno cada vez más dinámico y complejo.

\title{
Referencias
}

Asimov, I. (1942). Runaround. Astounding Science Fiction, 29(1), 94-103. Recuperado de http://www.isfdb.org/cgi-bin/title.cgi?44191 
Asimov, I. (1989). Círculo vicioso: los robots. Barcelona: Martínez Roca.

Broadband Commission for Sustainable Development. (2016). The state of broadband: Broadband catalyzing sustainable development. Recuperado de http://broadbandcommission.org/Documents/reports/ bb-annualreport2016.pdf

Carr, N. (2010). The shallows: What the internet is doing to our brains. Nueva York, EE. UU.: WW Norton y Company.

Carey, J. W. (1967). Harold Adams Innis and Marshall McLuhan. The Antioch Review, 27(1), 5-39. DOI: 10.2307/4610816

Christopherson, R. W. (1994). Geosystems: An introduction to physical geography. New Jersey, EE. UU.: Prentice Hall.

Darwin, C. (1859). On the origin of species by means of natural selection. Londres, EE. UU.: J. Murray.

DeAngelis, T. (2000). Is internet addiction real? Monitor on Psychology, 31(4). Recuperado de http://www.health.am/psy/more/is-internet-addiction-real/

Duffy, D. (1969). Marshall McLuhan. Toronto, Canadá: McClelland and Stewart.

Eddington, A. S. (1987). Space, time and gravitation: An outline of the general relativity theory. Cambridge, RU: Cambridge University Press.

Federman, M. \& De Kerckhove, D. (2003). McLuhan for managers. Canada: Viking.

Fidler, R. (1997). Mediamorphosis. Understanding new media. Thousand Oaks, EE. UU.: Pine Forge Press. 
Gordon, W. T. (1988). Marshall McLuhan. Escape into understanding: The authorized biography. Basic Books.

Gould, S. J. (1991). Exaptation: A crucial tool for evolutionary psychology. Journal of Social Issues, 47, 43-65. DOI: 10.1111/j.1540-4560.1991. tb01822.x

Gould, S. J. y Vrba, E. S. (1982). Exaptation: A missing term in the science of form. Paleobiology, 8(1), 4-15. DOI: https://faculty.insead.edu/ vibha-gaba/documents/Gould\%20\%26\%20Vrba_Exaptation.pdf

IAB México (2017, marzo 30). Estudio de consumo de medios y dispositivos entre internautas mexicanos 2017. Recuperado de http://www.iabmexico.com/estudios/consumo-medios-2017/

Innis, H. (1951). The bias of communication. Toronto, Canadá: University of Toronto Press.

Innis, H. (1972). Empire and communications. Toronto, Canadá: University of Toronto Press.

Levinson, P. (1999). Digital McLuhan: A guide to the information millennium. Nueva York, EE. UU.: Routledge.

Levinson, P. (2004). Cellphone: The story of the world's most mobile medium and how it has transformed everything. Nueva York, EE. UU.: Palgrave.

Logan, R. K. (2004) The alphabet effect: A media ecology understanding of the making of western civilization. Cresskill, EE. UU.: Hampton Press.

Marchand, P. (1989). Marshall McLuhan: The medium and the messenger. Nueva York, EE. UU.: Ticknor y Fields.

McLuhan. E. (2016). 50 años después... Retrospección y perspectiva de la obra de Marshall McLuhan. En O. Islas Carmona, F. Gutiérrez 
Cortés y L. Strate (Coords.), La comprensión de los medios en la era digital: un nuevo análisis de la obra de Marshall McLuhan. Ciudad de México, México: Alfaomega.

McLuhan, E. \& McLuhan, M. (2011). Theories of communication. Nueva York, EE. UU.: Peter Lang.

McLuhan, M. (1967). The medium is the massage: An inventory of effects. Nueva York, EE. UU.: Gingko Press.

McLuhan, M. (1969). Counterblast. Toronto, Canadá: McClelland and Stewart.

McLuhan, M. (1970). Culture is our Business. Nueva York, EE. UU.: McGraw-Hill.

McLuhan, M., McLuhan, M. A., \& Lapham, L. H. (1994). Understanding media: The extensions of man. Cambridge, EE. UU.: MIT Press.

McLuhan, M. (1996). Comprender los medios de comunicación: las extensiones del ser humano. Barcelona, España: Paidós.

McLuhan, M. (1998). La Galaxia Gutenberg: génesis del homo typographicus. Barcelona, España: Círculo de Lectores.

McLuhan, M. (2002). The mechanical bride: Folklore of industrial man. Nueva York, EE. UU.: Ginko Press.

McLuhan, M. (2004). Understanding me: Lectures and interviews. Cambridge, EE. UU.: The MIT Press.

McLuhan, M. (2005). Marshall McLuhan unbound. Nueva York, EE. UU.: Gingko Press.

McLuhan, M. \& Carpenter, E. (1970). Explorations in communications. Londres, RU: Cape. 
McLuhan, M. \& Carson, D. (2003). The book of probes. Nueva York, EE. UU.:: Ginko Press.

McLuhan, M. \& Fiore, Q. (1968). War and peace in the global village. Nueva York, EE. UU.: McGraw-Hill.

McLuhan, M. \& Fiore, Q. (1987). El medio es el masaje: un inventario de efectos. Barcelona, España: Paidós.

McLuhan, M. \& McLuhan, E. (1988). Laws of media: The new science. Toronto, Canadá: University of Toronto Press.

McLuhan, M. \& McLuhan, E. (2011). Media and formal cause. Houston, EE. UU.: NeoPoiesis Press.

McLuhan, M., McLuhan, E. \& Hutchcon, K. (1977). The city as classroom. Ontario, Canadá: Book Society of Canada.

McLuhan, M. \& Nevitt, B. (1972). Take today: The executive as dropout. Nueva York, EE. UU.: Harcourt Brace.

McLuhan, M. \& Parker, H. (1968). Through the vanishing point: Space in poetry and painting. Nueva York, EE. UU.: Harper.

McLuhan, M. \& Watson, W. (1970). From cliché to archetype. Nueva York, EE. UU.: Viking Press.

Media Ecology Association. Recuperado de http://www.media-ecology. org/media_ecology/

Miller, J. (1971). McLuhan. Nueva York, EE. UU.:: Viking Press.

Nystrom, C. (1973). Towards a science of media ecology: The formulation of integrated conceptual paradigms for the study of human communi- 
cation Systems (Tesis de doctorado, New York University, Nueva York, Estados Unidos).

Oliver, N. (2015, agosto 23). El móvil muda de piel. En El País. Recuperado de https://elpais.com/tecnologia/2015/08/18/ actualidad/1439918q462_506077.html

Ordóñez, J. (2003). Ciencia, tecnología e historia. Ciudad de México, México: Fondo de Cultura Económica.

Piscitelli, A. (2005). Internet: la imprenta del siglo XXI. Barcelona, España: Gedisa.

Postman, N. (1970). The reformed english curriculum. En A. C. Eurich (Ed.), High school 1980: The shape of the future in American secondary education (pp. 160-168). Nueva York, EE. UU.: Pitman.

Postman, N. (1979). Teaching as a conserving activity. Nueva York, EE. UU.:: Dell.

Postman, N. (1986). Amusing ourselves to death: Public discourse in the age of show business. Nueva York, EE. UU.:: Penguin.

Postman, N. (1993). Technopoly: The surrender of culture to technology. Nueva York, EE. UU.:: Vintage Books.

Postman, N. (1998). Five things we need to know about technological change. Conferencia dictada en Denver (Colorado). Recuperado de http://web.cs.ucdavis.edu/ rogaway/classes/188/materials/ postman.pdf

Postman, N. \& Weingartner, C. (1969). Teaching as a subversive activity. Nueva York, EE. UU.:: Delacorte.

Rees, F. (2006). Johannes Gutenberg, Inventor of the printing press. Minneapolis: Compass Point Books. 
Reynolds, G. (2007). An army of Davids: How markets and technology empower ordinary people to beat big media, big government, and other Goliaths. Nueva York, EE. UU.:: Thomas Nelson Inc.

Rheingold, H. (2013). Mind amplifier: Can our digital tools make us smarter? Ted Conferences.

Sánchez de Antuñano, J. (2014). Apuntes del seminario de investigación. Manuscrito no publicado, División de Ciencias y Artes para el Diseño, Universidad Autónoma Metropolitana, Unidad Azcapotzalco, México.

Toffler, A. y Toffler, H. (2006). La revolución de la riqueza. Barcelona, España: Debate.

World Economic Forum. (2017). The global competitiveness report 20162017. Recuperado de http://www3.weforum.org/docs/ GCR2016-2017/05FullReport/TheGlobalCompetitivenessReport2016-2017_FINAL.pdf 